\title{
Comprensión del modelo hereditario de Mendel tras la enseñanza habitual en alumnos de educación secundaria obligatoria
}

\section{Understanding of Mendelian Inheritance Model after conventional teaching methods in Secondary Education students}

\author{
José Antonio García Lillo \\ Patricia Quinto Medrano \\ Joaquín Martínez Torregrosa \\ Departamento de Didáctica General y Didácticas Específicas \\ Universidad de Alicante
}

\begin{abstract}
Resumen: Mostrar que una nueva propuesta de enseñanza produce mejores actitudes y aprendizajes en los alumnos requiere disponer de un análisis de lo que se hace y se consigue con la enseñanza habitual. Para realizar dicho análisis se ha efectuado un estudio histórico y epistemológico de la evolución de las ideas en genética clásica, identificando los problemas que están en su origen, las ideas que permitieron avanzar y los obstáculos que hubo que superar. Como resultado de dicho estudio se han seleccionado un conjunto de indicadores de aprendizaje que deberían manifestarse en aquellas personas que hubieran comprendido los aspectos esenciales del modelo de herencia mendeliana, que se imparte en $4^{\circ}$ de ESO. Dichos indicadores se han utilizado para analizar el aprendizaje tras la enseñanza convencional del tema. En este trabajo se presentan los resultados obtenidos que muestran las deficiencias más comunes y justifican la necesidad de una propuesta diferente.
\end{abstract}

Palabras clave: didáctica de las ciencias, enseñanza habitual, genética clásica, modelo hereditario de Mendel, enseñanza de las ciencias en la E.S.O.

Abstract: Showing that a new approach produces better learning and attitudes in students requires to have an analysis of what is done and what is achieved with conventional teaching. In order to perform such analysis, a historical and epistemological study of the evolution of ideas in classical genetics has been made, identifying problems at their source, the ideas that allowed progress and the obstacles that had to be overcome. As a result of this study, we selected a set of indicators of learning that should manifest themselves in those people who have understood the essential aspects of Mendelian inheritance model, which is taught in 4th of E.S.O. These indicators have been used to analyze the learning after conventional teaching of the subject. In this paper we present the results that show the most common deficiencies and justify the need for a different approach.

Keywords: science education, conventional teaching, classical genetics, Mendelian inheritance model, science teaching in secondary.

(Fecha de recepción: octubre, 2014, y de aceptación: mayo, 2015)

DOI: $10.7203 /$ DCES.29.4265 


\section{Introducción}

La genética es sin lugar a dudas la rama de la Biología que más ha avanzado y más se ha popularizado en las últimas décadas. El Proyecto Genoma Humano, los alimentos transgénicos, el uso del ADN en criminología o en las pruebas de paternidad y las posibilidades de obtener clones humanos, son algunos ejemplos de las muchas aplicaciones de la genética que aparecen frecuentemente en los medios de comunicación. Es incuestionable su interés cuando cada día surgen nuevas noticias sobre sus avances y sus repercusiones éticas y sociales. Precisamente debido a sus implicaciones socioculturales, los ciudadanos deben ser capaces de manejar estas informaciones para poder tomar parte activa en las discusiones que se generan en este ámbito. Pero asimilar la gran cantidad de información nueva que aporta la investigación en genética sólo es posible si se adquiere con comprensión un modelo básico en el que se puedan integrar todas estas nuevas aportaciones (Verhoeff, Jan Boerwinkel y Jan Waarlo, 2009). Por ello, es muy importante que el alumnado de enseñanza obligatoria no abandone las aulas sin haber conocido los principios elementales de la herencia de los caracteres biológicos y su modelo básico: el modelo de Mendel.

Además del claro interés que posee para lograr una correcta alfabetización científica del alumnado, a la genética se le ha prestado una gran atención debido a su importancia en la didáctica de la Biología (Bugallo-Rodríguez, 1995). Según Gagliardi (1986) los conceptos genéticos son conceptos estructurantes en la organización del conocimiento biológico, a partir de los cuales se pueden construir nuevos significados en temas como la reproducción, la evolución o la división celular. Además, la genética presenta gran interés educativo ya que permite aplicar estrategias de resolución de problemas, lo cual requiere el desarrollo de capacidades intelectuales y de hábitos de trabajo característicos de la actividad científica (Ayuso y Banet, 2002). Así, un uso funcional del modelo de Mendel para resolver problemas y hacer predicciones, supondría la adquisición de destrezas de alto nivel intelectual, aspiración de toda formación científica pero escasamente presentes en la enseñanza habitual de la Biología, en la que priman destrezas de bajo nivel intelectual como la memorización de datos y términos que después no se utilizan y se olvidan fácilmente (Momsen et al., 2010).

Se han realizado numerosas investigaciones en las que se ha puesto de manifiesto que los estudiantes tienen dificultades para entender muchos conceptos sobre genética y los mecanismos relacionados con la transmisión de la herencia biológica (Figini y De Micheli, 2005; Banet y Ayuso, 2003). A principios de los años ochenta la genética apareció entre los contenidos de Biología más importantes y más difíciles de aprender según los profesores de ciencias (Finley, Steward y Yarrowich, 1982) y, consecuentemente, surgieron 
diversas investigaciones sobre las dificultades de los alumnos y sus posibles causas. Entre ellas aparece el estudio de Bugallo-Rodríguez (1995) en el que se recogen una serie de preconcepciones y errores en los estudiantes derivados del uso de una terminología inadecuada y de la falta de relación entre conceptos clave; además esta autora destaca, coincidiendo con muchos otros autores, la resolución inadecuada y poco justificada de problemas. En un estudio más reciente, Caballero-Armenta (2008), seguía encontrando carencias similares en los alumnos sobre conceptos básicos de genética, la localización del material genético, su vía de transmisión o la reproducción sexual de las plantas; este autor detectó también deficiencias sobre conceptos básicos de probabilidad y el uso de variables estadísticas. Se hace por tanto necesario detectar no sólo los principales errores de comprensión de los estudiantes y los distintos obstáculos que se presentan en el aprendizaje de esta materia, sino también proponer metodologías de enseñanza innovadoras (Caballero et al., 1997) para alcanzar un aprendizaje significativo de la genética. El interés del aprendizaje de conocimientos genéticos en la enseñanza de las ciencias se ve reflejado en que Harlen (2010), en su libro de "Principios y Grandes Ideas de la Enseñanza de las Ciencias", identificaba la idea de que "la información genética se transmite de una generación a otra en los organismos" como una de las diez ideas clave a las que los alumnos se debían enfrentar en su educación en ciencias, para permitirles entender, disfrutar y maravillarse con el mundo natural.

En definitiva, la enseñanza-aprendizaje de la genética, parece plantear el mismo tipo de problemas encontrados al analizar el aprendizaje de otros temas dentro del modelo de enseñanza más habitual: la transmisión de conocimientos ya elaborados en su estado final. Estudios recientes coinciden en caracterizar la enseñanza habitual de la genética como un tipo de enseñanza básicamente transmisiva en el que se da mucho peso a la memorización de conceptos y menos importancia a la adquisición de procedimientos o actitudes científicas (Momsen et. al., 2010; Chavarría et al., 2012). Para intentar frenar esta tendencia en la enseñanza de las ciencias en la actualidad existe un amplio consenso entre los investigadores en didáctica, en que el aprendizaje y la enseñanza de las ciencias debe desarrollarse como un proceso de reconstrucción de conocimientos en un contexto de investigación (Verdú, 2004). En los grandes diseños curriculares, como los National Science Education Standards, se indica que "en todos los niveles, la educación científica debe basarse en la metodología de la investigación", como forma de favorecer, tanto una actividad significativa, en torno a problemas susceptibles de interesar a los estudiantes, como su progresiva autonomía de juicio y capacidad de participación en tareas colectivas (National Committee on Science Education Standards and Assessment, 1996). Además en un informe emitido por la Unión Europea, tras un análisis de la enseñanza 
donde se constata un descenso en el interés de los alumnos hacia las ciencias, también se aconseja una reorientación de su enseñanza hacia métodos basados en la investigación (Rocard et al., 2007). Dentro de esta orientación sobre la resolución de problemas de genética destacan los trabajos de Martínez-Aznar e Ibáñez-Orcajo (2005), coherentes con la propuesta metodológica de Gil y Martínez Torregrosa en los que proponen utilizar problemas totalmente abiertos, a modo de una verdadera investigación científica (Gil y Martínez Torregrosa, 1983). Planteando la enseñanza de esta forma encuentran un mayor uso de estrategias científicas en el alumnado, un pensamiento mejor estructurado sobre las ideas clave de la herencia y un cambio conceptual sobre la misma naturaleza de lo que es ciencia y lo que la ciencia hace. En este sentido algunos estudios recientes proponen salir al paso de la falta de interés de los alumnos hacia las ciencias mediante un enfoque histórico de las mismas, y concretamente proponen recurrir a aspectos históricos y epistemológicos en la enseñanza de la herencia biológica (Villa-García y TorresRosero, 2011; Mamlok-Naaman, 2011).

\section{Objetivos e hipótesis del trabajo}

Nuestro trabajo se inserta en una línea que pretende desarrollar toda la enseñanza de la Biología de un modo problematizado, por investigación guiada en torno a problemas fundamentales, de un modo similar a como se ha hecho en Física y en Química, ligando el estudio sobre las ideas y razonamientos erróneos a la planificación de la enseñanza (Martínez-Torregrosa y Verdú, 1993). En este modelo, la planificación de la enseñanza requiere de un protocolo que ha sido ampliamente contrastado en multitud de ocasiones (Verdú, 2004). Para planificar la enseñanza de un tema con estructura problematizada, el equipo de profesores/ investigadores necesita disponer de un conocimiento en profundidad de la materia a tratar, entendiendo por ello un conocimiento problematizado, consciente de cuáles fueron los problemas que están en el origen de los conocimientos en un campo determinado, cómo se ha llegado hasta ahí, cuáles fueron las dificultades que hubo que superar y las ideas que permitieron avanzar, el contexto social y las repercusiones tecnológicas que tuvieron y tienen los estudios en dicho campo, etc. (Osuna y col. 2007).

Esta planificación pasa por la realización de un estudio histórico y epistemológico de la evolución de las ideas en la genética clásica que permite identificar unos indicadores de comprensión del tema (ver Cuadro I) que servirán de referencia para los siguientes objetivos:

- Ver en qué medida dichos indicadores se alcanzan por los alumnos tras la enseñanza habitual del tema, y si los obstáculos previstos están presentes en ellos antes y después de la enseñanza.

- Identificar las ideas y razonamientos espontáneos que pueden dificultar el itinerario de avance previsto y que deben, por tanto, ser tenidos 
en cuenta en la planificación de la enseñanza.

- Servir de referencia para comparar los logros producidos mediante la enseñanza problematizada (cuando se ponga en práctica la secuencia de actividades en el aula) respecto a los de la enseñanza habitual.

Toda planificación debe ir acompañada de un análisis de la medida en que se consigue un aprendizaje con comprensión con la enseñanza habitual del tema. Si, con la enseñanza habitual, se consiguiera que un porcentaje importante de alumnos aprendiera con comprensión, tendría poco sentido un cambio didáctico. Además, dicho análisis también es necesario para una futura comparación de los logros (en conocimientos y actitudes) de los grupos experimentales con los logros que se obtienen habitualmente.

Así pues, la pregunta sobre la que vamos a tratar de obtener evidencia empírica es: ¿En qué medida se logra el aprendizaje con comprensión del modelo de transmisión hereditaria de Mendel tras la enseñanza habitual del tema en $4^{\circ}$ de ESO?

La hipótesis que ha guiado nuestro estudio es: Los alumnos de $4^{\circ}$ de ESO, después de la enseñanza habitual, no consiguen un aprendizaje con comprensión del modelo de Mendel.

La justificación de nuestra hipótesis se apoya en diferentes trabajos de investigación sobre las concepciones de los estudiantes en el campo de la genética que, aunque parciales, pueden ser interpretados como consecuencia de la existencia de obstáculos en su pensamiento, aún después de la instrucción sobre el tema, y la carencia de un modelo básico bien asumido (el de Mendel) que permita integrar los conocimientos sobre los genes. Véase por ejemplo los trabajos de Bugallo-Rodríguez (1995), Banet y Ayuso, (2003) o CaballeroArmenta (2008). Es necesario resaltar que, aunque estos resultados son indicadores claros de deficiencias en el aprendizaje, nuestra hipótesis es más global: se refiere a las deficiencias en los indicadores de comprensión de un modelo -el de Mendel- que es necesario para integrar jerárquicamente en él conocimientos más específicos sobre la genética moderna. No buscamos mostrar "errores conceptuales puntuales" sino la carencia en la adquisición de una estructura, un modelo, que dé sentido al aprendizaje sobre la herencia.

En este trabajo presentamos los resultados del análisis de la enseñanza convencional del modelo de herencia de Mendel en la etapa secundaria, cuya apropiación hemos considerado como el objetivo básico a conseguir por la enseñanza de la genética clásica en este nivel educativo. (Cuadro I)

\section{Metodología}

Elaboración del cuestionario y procedimiento para la recolección de datos

Teniendo en cuenta los indicadores de comprensión del modelo heredita- 


\section{Cuadro I}

Indicadores de comprensión para la enseñanza del modelo hereditario de Mendel en secundaria

1. Conocer los problemas que, en relación con las dificultades de predicción de caracteres heredables, están en el origen del modelo de Mendel, lo cual supone...

1.1.Ser capaces de describir el modo tradicional en que se ha intentado seleccionar y mantener razas de animales y variedades de plantas y describir su problemática asociada

1.2. Conocer las teorías existentes anteriores a Mendel y sus deficiencias en la predicción de caracteres heredables, como el modelo de herencia por mezcla de caracteres paternos

2. Apropiarse funcionalmente del modelo hereditario alternativo de Mendel en la búsqueda de regularidades, lo cual supone...

2.1. Comprender el papel del azar en la herencia independiente de los factores de un carácter físico, y para ello que:

- Un rasgo físico está controlado por la posesión de dos factores

- Estos factores se separan y solo se recibe uno de cada progenitor

- La expresión final del carácter depende de la combinación nueva que se genere tras la fecundación y la relación de dominancia entre ellos

2.2. Comprender el papel del azar en la herencia independiente de los factores de caracteres distintos, lo que a su vez implica entender que:

- La herencia de los factores de un carácter y su expresión no se ven influenciados por la herencia y expresión de otros caracteres

3. Conocer las limitaciones del modelo, cuando puede aplicarse (y cuando no) y ver la necesidad de un modelo más amplio.

rio de Mendel, hemos elaborado una serie de cuestiones para determinar en qué medida los alumnos, que han recibido enseñanza sobre el tema, consiguen dichos indicadores. Las cuestiones fueron discutidas con otros profesores/expertos de Biología, y distintas versiones del cuestionario se probaron con grupos piloto de alumnos de $4^{\circ}$ de
E.S.O., para seleccionar enunciados que no presentaban dificultades de comprensión y que permitían medir lo que buscábamos. La versión final del cuestionario se presenta en el Cuadro II, señalando el indicador que permite valorar cada una de las cuestiones.

Como es lógico, en la investigación en didáctica de las ciencias, la elabora- 
ción de cuestiones para indagar en la comprensión del contenido adquiere especial importancia. En nuestro caso, hemos procedido de la siguiente manera:

- En primer lugar, hemos tomado como "indicadores de comprensión" los grandes pasos necesarios para la comprensión del modelo de Mendel. Estos indicadores han sido puestos en común con un pequeño grupo de investigadores/profesores de Biología de Bachillerato que unánimemente los han reconocido como aquello que debería saber y saber hacer una persona que hubiera aprendido "de verdad", no repetitivamente, el modelo de Mendel.

- Sobre cada uno de los indicadores se han elaborado dos o tres cuestiones para indagar sobre su adquisición por los alumnos. Estas cuestiones han sido valoradas por tres profesores de Biología que han propuesto modificaciones o invalidado alguna de ellas.

- Con las cuestiones seleccionadas se han hecho pruebas-piloto con pequeños grupos de alumnos de $4^{\circ}$ curso de ESO (aprovechando el prácticum de estudiantes del Máster de Profesorado de Secundaria). En estas pruebas piloto se ha hablado con los alumnos para detectar dificultades con los enunciados (lo que ha servido para modificar algunas expresiones) y, particularmente, se han analizado sus respuestas para ver si se referían a la pregunta propuesta y si se podía sacar información sobre los indicadores de comprensión.

- Tras estas pruebas piloto se han seleccionado aquellas cuestiones que permitían extraer más información sobre los conocimientos de los alumnos.

A continuación (en el Cuadro II) se muestran las cuestiones seleccionadas junto con el indicador sobre el que permiten obtener información y se comenta de manera detallada qué debería contener una respuesta y carencias que esperamos encontrar.

Las dos primeras cuestiones se propusieron para obtener información sobre el conocimiento problematizado del modelo de Mendel que tienen los alumnos, es decir, acerca de los problemas que impulsaron a Mendel a elaborar su modelo para intentar darles respuesta (cuestión 1) y teorías alternativas a este modelo, ya fueran anteriores o coetáneas al mismo (cuestión 2). En ambas cuestiones no es preciso que el alumno explique ningún aspecto, sólo que cite los problemas o teorías que conozca que estuvieran en el origen del modelo de Mendel. Es verdad que un alumno puede comprender el modelo sin conocer cuales son los problemas que están en su origen $\mathrm{u}$ otras hipótesis diferentes, pero, sin duda, su comprensión no será de la misma "calidad" que si sabe, además, cuáles fueron los problemas que pudo solucionar y por qué fue aceptado frente a otros. Por lo tanto, si el alumno ha adquirido este primer gran paso a superar para la correcta comprensión del modelo debería ser capaz de iden- 


\section{Cuadro II \\ Cuestionario para el análisis del aprendizaje conseguido tras la enseñanza habitual del modelo de Mendel en la E.S.O.}

\section{Cuestiones}

Indicador de comprensión

Cuestión 1. Di problemas de interés (desde la antigüedad hasta la actualidad) que han podido ser solucionados según se ha avanzado en el conocimiento sobre la herencia.

1. Problemas en el origen del modelo y teorías alternativas

Cuestión 2. En clase has estudiado las ideas de Mendel sobre la herencia. Mendel vivió en el siglo XIX y sus ideas se aceptaron a principios del siglo XX. Cita alguna/s ideas o teorías sobre la herencia anteriores o distintas a las de Mendel.

Cuestión 3. Imagina que tienes que explicarle a una persona, que no ha estudiado Biología, las ideas importantes sobre la herencia (es decir sobre cómo se transmiten rasgos de los padres a los hijos) que has aprendido. ¿Qué le dirías?

2. Apropiación funcional del modelo de Mendel

Cuestión 4. Un agricultor quiere cultivar dos variedades de flores de una misma planta (flores rojas y flores blancas). Quiere dividir la plantación en dos invernaderos, uno que produzca solamente plantas con flores rojas y otro que produzca solamente plantas con flores blancas. Para ello compra en el vivero una sola planta roja y una sola planta blanca. Suponiendo que el color de la flor de dicha planta sigue una herencia mendeliana, elige razonadamente el mejor plan para ayudarle a conseguirlo.

Cuestión 5. Una industria agroalimentaria necesita un suministro continuo de guisantes amarillos y lisos para su envasado y venta posterior. Para ello establecieron una gran plantación de plantas de guisante, en un invernadero y bajo condiciones controladas; así cada planta puede originar decenas de semillas. Los guisantes que se utilizaron en la siembra fueron todos de la variedad deseada amarilla-lisa. Cuando crecieron las plantas se permitió su autofecundación (el polen de las flores de cada planta fecundó sus mismos óvulos, ya que en ellas los estambres y el pistilo permanecen muy cerca y están aislados del exterior por sus propios pétalos que los envuelven). Al llegar la recolección, en contra de lo esperado, se observaron tres variedades de plantas, según se describen a continuación:

a) Variedad 1: plantas donde todos los guisantes eran amarillos y lisos.

b) Variedad 2: plantas donde aparecían sobre todo guisantes amarillos y lisos pero también verdes y lisos.

c) Variedad 3: Plantas donde aparecían sobre todo guisantes amarillos y lisos, pero también verdes-lisos y amarillos-rugosos y en menor proporción verdes y rugosos.

Te pedimos que utilices lo que has aprendido del modelo de herencia de Mendel para explicar las causas de que haya ocurrido así.

Cuestión 6. El modelo de herencia de Mendel supuso la primera demostración de la existencia de reglas en la herencia de caracteres o rasgos físicos. ¿Crees que se cumple siempre? Explica con tus palabras cuando puede aplicarse y cuando no, es decir si se cumple de forma general o si hay casos en los que no se cumple.

3. Limitaciones de validez del modelo de Mendel. 
tificar problemas en el origen (cuestión 1) como la obtención y selección de variedades de animales y plantas o los estudios de enfermedades hereditarias; y de identificar teorías anteriores o alternativas al modelo de Mendel para la herencia de caracteres (cuestión 2) como las corrientes "preformacionista", la idea de la herencia de partículas asociadas con la sangre o el modelo de herencia por mezcla de caracteres de los antepasados de Galton y Pearson (Galton, 1889). Sin embargo, si el estudiante no ha comprendido ni adquirido este gran paso, no hará referencia a los problemas en el origen sino que citará problemas y aspectos relacionados con las aplicaciones actuales de la genética, que están mucho más al alcance de la cultura general por su gran presencia en los medios de comunicación; y en vez de teorías sobre la herencia de caracteres distintas a la de Mendel citará otras teorías no relacionadas directamente como la teoría de la evolución de Darwin o el fijismo, que pueden recordar puesto que son teorías que se estudian a lo largo del mismo curso escolar de $4^{\circ}$ ESO.

El segundo gran paso a superar es el de apropiarse funcionalmente del modelo de Mendel. Para analizar la apropiación funcional del modelo por parte de los estudiantes se han utilizado tres cuestiones (cuestiones 3,4, y 5) cuyas respuestas y la consistencia entre tales respuestas mostrarán el grado de apropiación del modelo. La cuestión 3 es de naturaleza abierta y pretende que el alumno explique con sus propias palabras las ideas importantes sobre la herencia, por tanto, que explique el modelo de Mendel. Una buena explicación del modelo que abarque todas sus ideas importantes (apartados 2.1 y 2.2 del Cuadro I) deja explícita la apropiación del mismo. Pero esta pregunta no es concluyente por sí sola puesto que un alumno puede haber comprendido el modelo pero no explicarlo adecuadamente por sus carencias en expresión escrita (algo habitual). Por eso esta cuestión 3 se apoya en las cuestiones 4 y 5 , de una naturaleza más "académica o prototípica”, es decir, más similares a los típicos problemas de genética de "papel y lápiz" utilizados en la enseñanza habitual. La cuestión 4 propone la resolución de un problema sobre la herencia de un carácter y la cuestión 5 para dos caracteres; en ambos casos, una resolución adecuada de estos problemas debería contener la expresión de ideas y razonamientos cualitativos sobre el modelo (o "las leyes") de Mendel, no sólo la nomenclatura y el procedimiento algorítmico habitual en la resolución. Un alumno que no haya comprendido el modelo de Mendel y no haya conseguido su apropiación funcional será incapaz de explicar las ideas importantes de la herencia en su conjunto (cuestión 3), y tan sólo dará pinceladas de las mismas o intentará explicar un ejemplo de herencia concreto (como la del color de los ojos o el pelo en humanos); y en la resolución de los problemas (cuestiones 4 y 5 ) utilizará una forma automática de resolución sin ofrecer una explicación al problema que deje ver que ha entendido el mode- 
lo de Mendel adecuadamente. En cualquier caso, esperamos conseguir mejores resultados en las cuestiones 4 y 5 que en la cuestión 3 , debido a la dificultad adicional de la cuestión 3 que precisa de una buena expresión escrita, y a que las cuestiones 4 y 5 son similares a las cuestiones que puedan haber trabajado en las aulas, aunque expresadas de una forma más abierta que los problemas habituales.

El tercer indicador, acerca del conocimiento de las limitaciones de validez del modelo de Mendel, que abre paso a futuros temas como la teoría cromosómica de la herencia, se analiza mediante la cuestión 6. En ésta se pregunta directamente sobre el conocimiento de estas limitaciones, por lo que un alumno que haya comprendido bien el modelo de Mendel se referirá a la existencia de caracteres diferentes que se hereden conjuntamente (en contra de la independencia total hipotetizada por Mendel), y que no cumplen, por tanto, cuantitativamente las proporciones que se deducen del modelo; mientras que un alumno que no lo haya comprendido adecuadamente pensará que el modelo es universal o como mucho se referirá a casos en los que sí se cumplen las leyes de la herencia mendeliana pero se desvían de las proporciones fenotípicas habituales, como los casos de codominancia, herencia intermedia o alelismo múltiple; la alusión a estos nuevos casos se han tomado como una "profundización" del modelo inicial y no como una verdadera limitación del modelo, ya que en ellos no varía el "mecanismo azaroso" de la independen- cia, sino el número de las piezas que se combinan al azar (alelismo múltiple) o la relación entre ellos (codominancia o herencia intermedia).

De este modo elaboramos un instrumento formado por las seis cuestiones anteriores. El tiempo para responder con tranquilidad se calculó en unos 50 minutos, es decir, su cumplimentación ocuparía el desarrollo de una sesión. La respuesta debía ser individual y anónima, y realizada después de haber tratado el tema sobre la herencia que se encuentra en el programa de Biología de $4^{\circ}$ de ESO. En la práctica, la inmensa mayoría de los alumnos requirió menos tiempo del previsto.

Dada la dificultad evidente para elegir grupos por muestreo aleatorio, aprovechamos el Prácticum de alumnos del Máster de Profesorado de Secundaria y enviamos un correo electrónico a numerosos profesores de Biología y Geología de institutos, para explicarles la finalidad de nuestro trabajo y solicitarles su colaboración para pasar el cuestionario a sus alumnos. De este modo, totalmente voluntario, conseguimos 297 alumnos pertenecientes a 12 institutos de secundaria repartidos por la provincia de Alicante. Se trata, pues, de un estudio cuasi experimental.

En cuanto al procedimiento seguido, el cuestionario se repartió a los profesores de Biología y Geología que impartían el nivel de $4^{\circ}$ ESO durante el curso 2012-2013 en distintos centros de enseñanza secundaria de la provincia de Alicante. Estos cuestionarios fueron enviados y recibidos por correo, y los encarga- 
dos de pasarlos a los alumnos fueron sus propios profesores, lo cual, en todo caso, podría generar resultados más desfavorables para nuestra hipótesis (cabe la posibilidad de que se pudiera dar el caso de repaso del tema antes de la aplicación del cuestionario; de ayudas y orientaciones de los profesores en el transcurso del mismo; o, simplemente, de enviárnoslo sólo si "salía bien"). En cualquier caso, la aplicación de dicho instrumento fue individual durante el desarrollo de una clase y se llevó a cabo en horario escolar en una sesión de al menos 50 minutos de la materia de Biología y Geología de $4^{\circ}$ ESO, previa explicación de las instrucciones a los alumnos. Los cuestionarios se pasaron tras la enseñanza habitual de la genética clásica y herencia mendeliana, variando en todos los casos el tiempo transcurrido entre la enseñanza del tema y la aplicación del cuestionario, desde tan sólo unas sesiones de demora hasta transcurridas tres semanas.

\section{Procedimiento de interpretación y valoración de las respuestas al cuestionario}

Al tratarse de un cuestionario de respuesta abierta, el análisis del contenido de las respuestas adquiere toda la relevancia y, al mismo tiempo, plantea problemas de fiabilidad. Estos problemas han sido abordados y resueltos, en el campo de la investigación en didáctica de las ciencias, mediante la elaboración de rúbricas y redes de análisis, y el seguimiento de un protocolo de valoración explícito por, al menos, dos exper- tos (en este caso dos profesores e investigadores en didáctica de la Biología). Todo este proceso debe ir acompañado, además, con la exposición de ejemplos concretos de respuestas de alumnos en los que se describa cómo se ha llegado a la valoración otorgada.

En este estudio, la forma de proceder ha sido:

1. Elaboración de una red de análisis (Anexo 1) para la respuesta de cada una de las cuestiones. Esta red de análisis ha sido elaborada por tres expertos (uno de ellos Doctor en Biología, profesor de Biología y profesor de Didáctica de las Ciencias, otro Investigador y Catedrático de Didáctica de las Ciencias y, por último, una profesora de Biología e investigadora en Didáctica de las Ciencias, los autores del trabajo), y según se ha ido utilizando en el análisis de las respuestas, se han ido añadiendo ítems (pocos) no contemplados inicialmente para poder categorizar el máximo número de ideas (correctas o no) que aparecían en ellas.

2. Una vez analizada cualitativamente una respuesta a cada una de las cuestiones del instrumento utilizado, se ha asignado un valor de 0,1 o 2 para expresar el grado de consecución del indicador de comprensión correspondiente ( 0 = no conseguido; 1 = conseguido parcialmente; 2 = conseguido). Es necesario resaltar que se ha hecho explícita la relación entre las ideas que se expresaban o que faltaban en las respuestas y la puntuación otorgada, de manera que se pudiera traducir el significado de dicha puntuación en qué es lo que sabía o no sabía cada uno de los alumnos. 
3. Inicialmente, cada uno de los dos investigadores biólogos ha valorado, con el estadillo descrito, diez cuestionarios (los mismos) extraídos al azar entre los cuestionarios recibidos. Se ha realizado una puesta en común, en la que se ha discutido algún caso discrepante y se han añadido descriptores para valorar las respuestas. Con ello, se ha mejorado el estadillo inicial y con el nuevo estadi- llo se han valorado, independientemente, veinte nuevos cuestionarios. Tras la nueva puesta en común hemos encontrado más del $90 \%$ de coincidencia en las valoraciones, por lo que consideramos validado el instrumento. A partir de ahí se procedió a la valoración del resto de cuestionarios $(n=297)$.

En el Cuadro III, a modo de ejemplo, aparece cómo se ha analizado la cues-

\section{Cuadro III. Criterios de valoración de las respuestas para la cuestión 1 y ejemplos de respuestas representativas de cada valoración}

$$
\text { ( } 0 \text { = no conseguido; } 1 \text { = conseguido parcialmente; } 2 \text { = conseguido) }
$$

Cuestión 1. Acerca de problemas que han podido ser solucionados con conocimientos sobre la herencia y que están en el origen del modelo.

2 El alumno señala problemas sobre obtención de variedades de plantas y animales con rasgos físicos deseados y estables y problemas asociados a la aparición de enfermedades heredables.

1 Señala sólo uno de los aspectos anteriores (mayoritariamente enfermedades).

0 No hace referencia a ninguno de estos problemas o alude a resolución de problemas posteriores, relacionados con aplicaciones de ingeniería genética, o respuesta en blanco.

Ejemplo de Respuesta tipo 2 a Cuestión 1. Se refiere a los dos principales problemas que están en el origen del modelo de Mendel

Ejemplo de Respuesta tipo 1 a Cuestión 1. Se refiere a tan solo un problema, mayoritariamente las enfermedades hereditarias

Ejemplos de Respuesta tipo 0 a Cuestion 1. Se refieren a aplicaciones de la genttica moderna

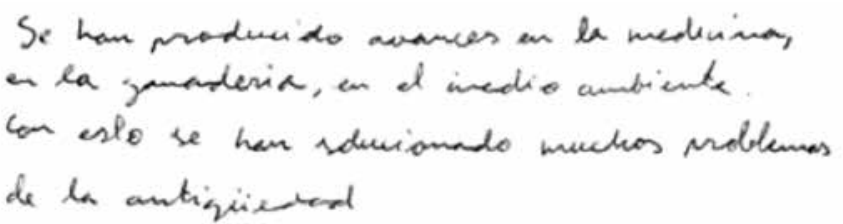

Por ejenplo: Eofermedaneo.

Hay enfermedadts que don heretabers, juo no per dfto tes hijos ticuer que padecrese.

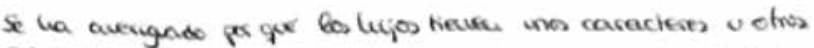

Ei: pacte gos marrones, macre gos masrones. hijo ops a tufes.

Se saber les grupos senguiess, sus antigeos y articuecpos para poder tronsferir sangre sun que as pase rade.

Se pucke siber si un niño que esta on el leto tedia malforma. cones y ar de suien biva hederado

Comprober s: toll $h_{i j}$ es to vontulios $h_{j}$ o

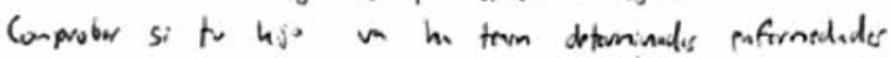


tión 1, con la que se estudia parte del grado de adquisición del indicador 1 (véase Cuadro II), junto a ejemplos representativos de respuestas dadas. En el Anexo 1, mostramos el resto de la red de análisis (tras su utilización en los primeros diez cuestionarios) y nuevos ejemplos para cada cuestión sobre cómo se han valorado las diferentes respuestas de los alumnos.

Para la estructuración, ordenación y análisis de los datos se utilizó el paque- te de programas estadísticos Statistical Package for Social Sciences (SPSS Statistics) versión 21 para MacOS.

\section{Resultados}

Análisis descriptivo de las preguntas del cuestionario y del logro en los indicadores de comprensión

En el Cuadro IV se presentan los resultados de los alumnos sobre el conocimiento de problemas que están en

\section{Cuadro IV. Resultados sobre el conocimiento de los problemas que están en el origen de la invención del modelo de Mendel indicador de comprensión 1)}

Acerca de problemas que han podido ser solucionados con conocimientos sobre la herencia y que están en el origen del modelo (Cuestión $1 ; n=297$ )

Porcentaje de alumnos que señalan:

- Problemas sobre la obtención de variedades de plantas y animales con rasgos físicos deseados y estables y problemas asociados a la aparición de enfermedades heredables.

- Sólo uno de los aspectos anteriores (mayoritariamente enfermedades).

- No hace referencia a ninguno de estos problemas o alude a resolución de problemas posteriores relacionados con aplicaciones de ingeniería genética o dejan su respuesta en blanco.

Acerca de las teorías de la herencia anteriores o distintas a la de Mendel (Cuestión 2; n = 297)

Porcentaje de alumnos que hacen referencia a:

- Teorías sobre la herencia anteriores o distintas (teorías preformacionistas y epigenéticas de la herencia o teoría de la herencia de Galton como mezcla proporcional de caracteres de los progenitores y antepasados)

- Otras teorías biológicas no relacionadas directamente con la herencia de caracteres (teoría de la evolución de Darwin, la de los caracteres adquiridos de Lamarck, fijismo, creacionismo, etc.) o no hace referencia a teoría alguna (respuesta en blanco)

Grado de adquisición del indicador de comprensión 1 ("Conocer los problemas que, en relación con las dificultades de predicción de caracteres heredables, están en el origen del modelo de Mendel”)

- Total (expresan problemas de variedades de animales y plantas y enfermedades heredables y conocen algún intento de teorizar sobre ellos).

- Parcial (expresan más de un problema en el origen pero no conocen hipótesis o teorías anteriores o distintas).

- No adquirido (como máximo hacen referencia solamente a un problema). 
el origen de los trabajos de Mendel y teorías anteriores o que competían con la mendeliana (lo que constituye el primer indicador de comprensión).

La información necesaria para interpretar los resultados se encuentra en la misma tabla. Como puede verse, el $97 \%$ de los alumnos de la muestra estudiada $(\mathrm{n}=297)$ no dispone de un conocimiento problematizado en genética clásica sobre el modelo de Mendel, pues no es capaz de expresar los problemas cuyo intento de solución y control ha dado lugar a la existencia y evolución de las ideas científicas sobre la herencia. No conocen tampoco (jaunque muchos de ellos tienen ideas muy cercanas a la de que la herencia transmitida es una mezcla de los rasgos de padres y antecesores!) hipótesis y teorías anteriores o simultáneas a la de Mendel. Como mucho, mencionan otras teorías biológicas que no están relacionadas directamente con la herencia, pero que se estudian durante el mismo curso escolar, como la teoría de la evolución de Darwin o el fijismo o el creacionismo. Tan sólo 9 de los 297 alumnos (3\%) nombran los dos principales problemas que están en el origen del modelo de Mendel como son la obtención de variedades de plantas y animales con rasgos deseados y estables y la aparición de enfermedades hereditarias. Del resto de alumnos, el $29,3 \%$ sólo hace referencia a las "enfermedades" (muchas veces sin incluir esa palabra en una frase que exprese una idea) como uno de los problemas que han podido ser "resueltos" mediante conocimientos sobre la herencia. Pero la mayoría de ellos, el 67,7\%, desconoce los problemas que están en el origen del modelo y sólo citan problemas relacionados con la ingeniería genética y sus aplicaciones más resaltados por los medios de comunicación, como tratamiento de enfermedades con terapia génica o alimentos transgénicos. Estos resultados tan rotundos muestran que el aprendizaje (iy la enseñanza!) se ha producido de una forma aproblemática y ahistórica.

Teniendo en mente que se trata de un estudio cuasiexperimental, la estimación del error típico de la población es de un $1 \%$. El intervalo de confianza del $95 \%$ es $0,95<\pi<0,99$. Eso significa que existe una probabilidad del $95 \%$ de que la proporción de alumnos de $4^{\circ}$ de $\mathrm{ESO}$, que no conocen los problemas que están en el origen del modelo de Mendel y teorías anteriores o simultáneas sobre la herencia, esté comprendida en dicho intervalo.

A continuación, en el Cuadro V, se muestran los resultados respecto a la existencia y persistencia de obstáculos para la apropiación funcional del modelo de Mendel, que constituye el segundo indicador de comprensión. Analizando estos resultados, podemos afirmar que un $84,2 \%$ de los alumnos no ha adquirido un aprendizaje funcional del modelo de Mendel. En este caso, el intervalo de confianza del $95 \%$ es $0,802<\pi$ $<0,882$, lo que significa que existe una probabilidad del $95 \%$ de que el intervalo incluya el valor de la proporción en la población. Para valorar el grado de adquisición de este segundo indicador 


\section{Cuadro V. Resultados sobre la existencia y persistencia de obstáculos para la apropiación funcional del modelo de Mendel indicador de comprensión 2)}

Sobre la utilización con sentido de las hipótesis del modelo de Mendel cuando tratan de explicar a otra persona las ideas importantes sobre la herencia (Cuestión $3 ; \mathrm{n}=297$ )

Porcentaje de alumnos que cuando tratan de explicar las ideas importantes sobre la herencia a otra persona:

- Expresan los aspectos básicos de la teoría mendeliana (que un rasgo físico está controlado por la posesión de dos factores (1), que éstos necesariamente se han de separar (2), que en la descendencia sólo se recibe uno de cada progenitor (3) y que tras la fecundación pueden surgir combinaciones de parejas de factores distintas a las paternas (4), por lo que la expresión final del carácter dependerá de la combinación que se genere y de la relación de dominancia entre ellos(5)) (Adquisición total)

- No expresan todos los aspectos anteriores (o lo hacen inadecuadamente), o utilizan directamente un ejemplo concreto con la simbología típica de alelos o factores, sin acompañamiento de explicación abstracta (Adquisición parcial)

- No se refiere a aspectos básicos concretos de la herencia mendeliana (como cuando expresan sólo que la mitad del $\mathrm{ADN}$ proviene del padre y la otra de la madre, que las informaciones genéticas del padre y de la madre "se mezclan") o aluden a términos relacionados (como "genes dominantes y recesivos" o la herencia ligada al sexo) pero sin explicar en ningún caso cómo se transmiten. (No adquirido)

Sobre la utilización del modelo de Mendel en la resolución de un problema (Cuestión 4; n = 297) Porcentaje de alumnos que al planificar una estrategia para conseguir dos poblaciones con variedades estables de un carácter, a partir de progenitores de genotipo desconocido, hacen uso del modelo de Mendel:

- Adecuadamente, haciendo referencia a las leyes del modelo para la herencia de un carácter (las indicadas en el apartado anterior de adquisición total), utilizando la simbología adecuada y explicando con las leyes mendelianas las proporciones que aparecen en la descendencia.

- Con deficiencias o de forma parcial, o utiliza la simbología mendeliana para un carácter pero sin argumentación alguna, o sólo indican la necesidad de averiguar si son variedades puras y utilizar la autofecundación pero sin desarrollar las leyes mendelianas ni utilizar la simbología característica.

- Erróneamente (no saben utilizar funcionalmente el modelo de Mendel), o alude a términos y/o metodologías posteriores al modelo, o no utiliza la simbología ni la argumenta adecuadamente, o mencionan o identifican la ley de Mendel a la que hac referencia la cuestión pero no la aplican para dar respuesta al problema, o dejan la respuesta en blanco.

$161(54,2 \%)$

Sobre la utilización del modelo de Mendel en la resolución de un problema (Cuestión 5; n = 297) Porcentaje de alumnos que al intentar explicar los resultados de un estudio empírico sobre la herencia de dos caracteres, con la desaparición y reaparición de variedades de caracteres en generaciones distintas, hacen uso del modelo de Mendel:

- Adecuadamente, haciendo referencia a las leyes del modelo para la herencia de dos caracteres (las indicadas en el apartado de adquisición total de la primera celda de esta tabla y considerando que (6) la herencia de los factores de un carácter y su expresión no se ven influenciados por la herencia y expresión de otros caracteres), utilizando la simbología adecuada y explicando con las leyes mendelianas las proporciones surgidas en la descendencia. 
- Con deficiencias o de forma parcial, o utiliza la simbología mendeliana para dos caracteres pero sin argumentación alguna, o sólo indican la necesidad de aplicar la ley de Mendel relacionada con la herencia de dos caracteres de segregación independiente o la necesidad de parentales dihíbridos, pero sin desarrollar las leyes mendelianas ni utilizar la simbología característica.

- Erróneamente (no saben utilizar funcionalmente el modelo de Mendel), o alude a términos y/o metodologías posteriores al modelo, o no utiliza la simbología ni la argumenta adecuadamente, o mencionan o identifican la ley de Mendel a la que hace referencia la cuestión pero no la aplican para dar respuesta al problema, o dejan la respuesta en blanco.

Grado de adquisición del indicador de comprensión 2 ("Apropiación funcional del modelo hereditario de Mendel en la búsqueda de regularidades")

- Total (explican las ideas importantes sobre la herencia adecuadamente y desarrollan los problemas de herencia de uno y dos caracteres correctamente, explicando las leyes mendelianas para cada ejemplo y utilizando la simbología adecuada).

- Parcial (no explican adecuadamente las ideas importantes de la herencia pero sí plican correctamente el modelo en un ejemplo concreto; o lo explican adecuadamente pero no lo aplican correctamente en la resolución de problemas.

- No adquirido (como máximo utilizan la simbología adecuada pero sin argumentación alguna o identifican las leyes involucradas sin explicarlas ni aplicarlas en la resolución de problemas y no explican adecuadamente las ideas importantes sobre la herencia).

se han tenido en cuenta las respuestas a las cuestiones 3,4 y 5 y la coherencia entre ellas.

Cuando los alumnos tratan de explicar a otra persona las ideas más importantes sobre la herencia, y por tanto las hipótesis del modelo de Mendel (cuestión 3), tan sólo 5 alumnos de los 297 $(1,7 \%)$ lo hacen de una forma completa, expresando sus ideas básicas. Tan solo 72 alumnos $(24,2 \%)$ explican la herencia parcialmente o inadecuadamente: gran parte de ellos explican la herencia mediante un ejemplo (en muchos casos la herencia del color de los ojos en humanos) y muchos utilizan la simbología típica mendeliana pero sin acompañarla de una explicación adecuada. Pero el $74,1 \%$ de los alumnos (220 de los 297) no explican los aspectos básicos de la he- rencia, reduciendo su respuesta a que la información genética del padre y de la madre "se mezcla" para dar la información genética del hijo, o sólo citan la existencia de alelos dominantes y recesivos pero sin explicar cómo se transmiten.

Sin embargo, los porcentajes de adquisición parcial son relativamente elevados en las cuestiones 4 y 5 comparados con los de la cuestión 3 (alrededor del $42 \%$ de alumnos aplican el modelo de forma parcial en cuestiones 4 y 5 , frente al $24,2 \%$ que lo explican de forma parcial en cuestión 3), ya que se trata de dos problemas muy cercanos a los prototípicos que se resuelven en clase, lo que ha favorecido la repetición de rutinas y simbología, y porque en caso de duda siempre se ha actuado en contra de nuestra hipótesis en 
la valoración. Cuando se enfrentan a la resolución de problemas, el 3\% de los alumnos hacen uso del modelo de Mendel adecuadamente (utilizando la simbología adecuada y explicando con las leyes mendelianas las proporciones que aparecen en la descendencia) para la herencia de un carácter, y el 4,7\% de los alumnos usan el modelo adecuadamente para la herencia de dos caracteres. Hacen un uso del modelo con deficiencias o de forma parcial en un 42,8\% de los casos para la herencia de un carácter, y en el 41,1\% de las respuestas sobre herencia de dos caracteres (son casos que o utilizan la simbología mendeliana adecuadamente pero no argumentan la respuesta, o argumentan la respuesta indicando la/s ley/es de Mendel relacionada/s con el caso pero sin desarrollarla/s y sin utilizar la simbología característica). Tanto en herencia de uno como de dos caracteres, el 54,2\% de los alumnos hacen uso erróneo del modelo de Mendel (sin utilizar la simbología ni argumentar adecuadamente, o tan sólo mencionando la ley de Mendel a la que hace referencia la cuestión pero sin aplicarla para responderla).

Aun así, el aspecto más importante para valorar el grado de adquisición del indicador 2 sobre la apropiación funcional del modelo de Mendel es la consistencia de las respuestas a las tres cuestiones. Cuando se analiza la consistencia de lo que hacen en ambos problemas y lo que dirían a otra persona para explicarle las ideas importantes sobre la herencia (cuestión 3), el porcentaje de adquisición parcial baja mucho $(13,8 \%)$, y sólo un $2 \%$ de los alumnos (6 de 297) muestran un claro dominio del modelo mendeliano. El 84,2\% de los alumnos no alcanza una apropiación funcional del modelo, y como máximo utilizan la simbología adecuada pero sin argumentación alguna o identifican las leyes involucradas sin explicarlas ni aplicarlas en la resolución de problemas y en ningún caso explican adecuadamente las ideas importantes sobre la herencia. Esto confirma la presuposición de que gran parte de los alumnos realizan los ejercicios de genética de una forma mecánica y algorítmica pero sin comprender significativamente de un modo cualitativo/ abstracto la herencia de caracteres.

En cuanto a los resultados obtenidos para el tercer indicador de comprensión, como muestra el Cuadro VI anterior, la gran mayoría de los alumnos desconocen las limitaciones del modelo de Mendel tales como la existencia de caracteres cuya herencia no sigue lo que sería esperable según las hipótesis mendelianas. Tan sólo 18 alumnos $(6,1 \%)$ conocen las limitaciones del modelo de Mendel en casos de dependencia en la herencia de algunos caracteres cuyos genes están localizados en el mismo cromosoma. El 12,5\% de los alumnos (37) consideran que no se puede aplicar el modelo de Mendel a casos que realmente sí que cumplen la herencia mendeliana, pero en los cuales las proporciones fenotípicas de la descendencia varían, al ser casos especiales de relaciones de dominancia (como codominancia, herencia intermedia 0 herencia ligada al sexo entre otros). En 


\section{Cuadro VI. Resultados sobre el conocimiento de las limitaciones del modelo de Mendel y la necesidad de un modelo más amplio (indicador de comprensión 3)}

Grado de adquisición del indicador de comprensión 3 ("Conocer las limitaciones del modelo, cuando puede aplicarse (y cuando no) y ver la necesidad de un modelo más amplio") (Cuestión $6 ; \mathrm{n}=297$ )

- Total (hacen referencia de forma directa o indirecta al ligamiento de factores 0 dependencia en la herencia de caracteres, aludiendo a la localización sobre un mismo cromosoma).

- Parcial (no hacen referencia al ligamiento y aluden a casos de herencia mendeliana donde las proporciones típicas mendelianas "3:1" y "9:3:3:1" aparecen enmascaradas, como en los casos de codominancia, herencia intermedia, herencia ligada al sexo, genes letales, epistasias u otros casos).

- No adquirido (piensan que el modelo de herencia mendeliano es universal y se aplica siempre, o indican que no siempre se cumple pero sin argumentaciones o dejan la respuesta en blanco).

estos casos la diferencia aparece a la hora de manifestar en el fenotipo la herencia recibida de los progenitores, no en el proceso aleatorio de combinación de factores. Esto se considera como una adquisición parcial del conocimiento de las limitaciones del modelo de Mendel, puesto que son casos especiales en los que sí se cumple el modelo de Mendel pero se desvían de los ejemplos mendelianos en cuanto a las proporciones fenotípicas esperadas. Los 242 alumnos restantes $(81,4 \%)$ piensan que el modelo de herencia es universal y se aplica siempre, por lo que podemos afirmar que el porcentaje de alumnos de $4^{\circ}$ de ESO que desconoce las limitaciones del modelo de Mendel estará dentro del intervalo $0,770<\pi<0,858$ con una probabilidad del $95 \%$. Es necesario resaltar que estos alumnos, más de tres cuartas partes del total, están aprendiendo la genética moderna sin poderla integrar en el modelo básico de la herencia que es el mendeliano y sin poder advertir, por tanto, el avance que supuso para explicar anomalías y desviaciones que presentaba dicho modelo. El éxito del modelo de Mendel guió la moderna genética, pues se buscó la forma de explicar tanto su éxito como aquéllos casos en que no concordaba con los resultados experimentales.

En las figuras siguientes se muestran de forma resumida los resultados de las respuestas al cuestionario planteado, agrupando las respuestas en los tres niveles de valoración señalados anteriormente (Figura I), así como un análisis del nivel de adquisición de los indicadores de comprensión para el tema (Figura II).

A modo de resumen, los datos obtenidos en esta investigación revelan carencias muy rotundas en todos los indicadores de comprensión del modelo de herencia mendeliano y apoyan la relevancia de las metas y obstáculos previstos a 
Figura I. Análisis de las respuestas al cuestionario sobre la herencia mendeliana tras la enseñanza habitual

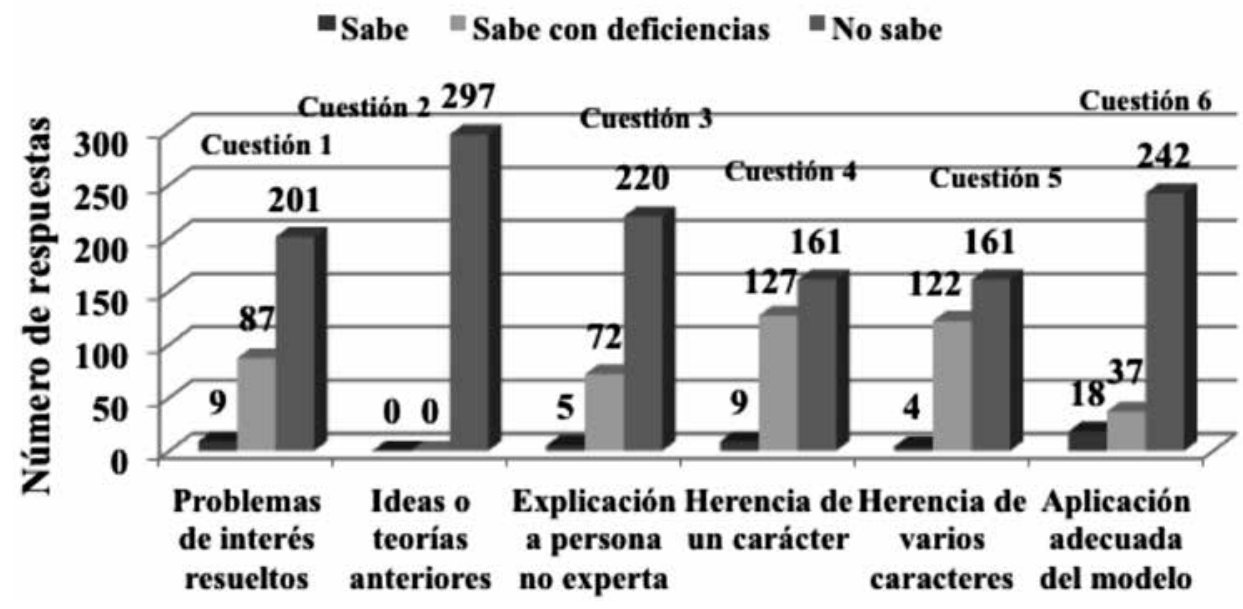

Figura II. Nivel de adquisición de los indicadores de comprensión para el modelo hereditario de Mendel

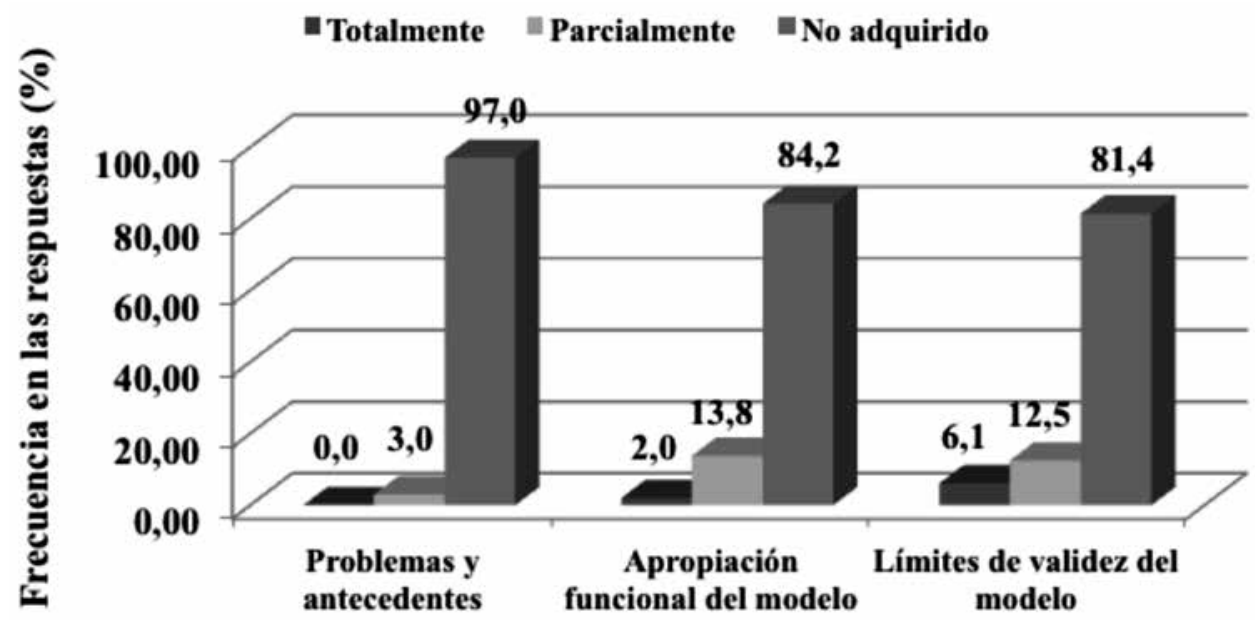

partir del estudio histórico y epistemológico sobre la evolución de las ideas de la genética clásica. Podemos afirmar que tras la enseñanza habitual de la genéti- ca clásica persisten estos obstáculos en los estudiantes de $4^{\circ}$ ESO:

- Desconocen los problemas y las hipótesis distintas que están en el 
origen del modelo de Mendel, dando lugar a una ciencia descontextualizada y aproblemática que dificulta la comprensión de qué es la ciencia y cómo se hace, así como un aprendizaje flexible y condicional.

- No se apropian funcionalmente del modelo, resolviendo los problemas de genética en muchos casos con la mera aplicación de un algoritmo a casos concretos.

- No conocen las limitaciones del modelo que hicieron necesaria su modificación y ampliación, lo que dificulta el interés y la capacidad de comprensión de otros aspectos de la genética más complejos como la teoría cromosómica, la genética molecular y la ingeniería genética.

\section{Conclusiones y perspectivas}

Los datos obtenidos en esta investigación, tal y como hemos analizado en el apartado de resultados, revelan carencias muy rotundas en todos los indicadores de comprensión del modelo de herencia mendeliano, confirmando la hipótesis que ha vertebrado este trabajo así como la relevancia didáctica potencial del cambio propuesto basado en la planificación de la enseñanza del tema desde el modelo de investigación guiada. Dicho de otro modo: hemos encontrado suficientes evidencias que apoyan nuestra hipótesis de que con la enseñanza habitual no se consigue el aprendizaje con comprensión, funcional, del modelo de Mendel. Y las deficiencias son tan importantes que existe mucho margen de mejora en la enseñanza/aprendizaje de este tema.

No obstante, en este trabajo -necesariamente limitado en el tiempo- no hemos desarrollado con detalle los obstáculos y/o razonamientos erróneos más frecuentes en nuestros alumnos (algo para lo que se necesita entrevistas orales que complementen los cuestionarios). Este conocimiento (sobre el que la investigación didáctica ha trabajado bastante) es imprescindible para concretar la enseñanza en estructura problematizada.

La elaboración, puesta en práctica en el aula (al menos en tres ocasiones) y evaluación de los conocimientos y actitudes generados en nuestros alumnos es el paso siguiente que debe ser abordado. Los resultados obtenidos en este trabajo serán útiles, además, para servir como grupo de control para comparar los resultados obtenidos en los grupos experimentales.

Por último, este estudio puede utilizarse en cursos de formación de profesores de Biología (inicial y en activo), para que tomen conciencia de las carencias de la enseñanza habitual y aprecien modelos de enseñanza alternativos que mejoren la alfabetización científica de nuestros alumnos.

\section{Referencias bibliográficas}

AYUSO, G. E. y BANET, E. (2002). Alternativas a la Enseñanza de la Genética en Educación Secundaria. Enseñanza de las Ciencias, 20 (1), 133-157. 
BANET, E. y AYUSO, G. E. (2003). Teaching of biological inheritance and evolution of living beings in secondary school. International Journal of Science Education, 25 (3), 373-407. DOI: 10.1080/09500690210145716.

BUGALLO-RODRÍGUEZ, A. (1995). La Didáctica de la genética: Revisión Bibliográfica. Enseñanza de las Ciencias, 13 (3), 379-385.

CABALLERO-ARMENTA, M. (2008). Algunas ideas del alumnado de secundaria sobre conceptos básicos de Genética. Enseñanza de las Ciencias, 26 (2), 227-244.

CABALLERO M., GONZÁLEZ, M. P., OLIVARES, E., SANTISTEBAN, A. y SERRANO, P. (1997). Didáctica de las Leyes de Mendel. Cuadernos de la UNED. Madrid: UNED.

CHAVARRÍA, S., BERMÚDEZ, T., VILLALOBOS, N. y MORERA B. (2012). El modelo Bandler-Grindler de aprendizaje y la enseñanza de genética mendeliana en estudiantes costarricenses de décimo año. Cuadernos de Investigación UNED, 4 (2), 213-221.

FIGINI, E. y DE MICHELI, A. (2005). La enseñanza de la genética en el nivel medio y la educación polimodal: Contenidos conceptuales en las actividades de los libros de texto. Enseñanza de las ciencias, Número extra. VII congreso.

FINLEY, F.N., STEWARD, J. H. y YARROWICH, W. I. (1982). Teacher's perceptions of important and difficult science content. Science Education, 66 (4), 531-538.

GAGLIARDI, R. (1986). Los conceptos estructurantes en el aprendizaje de la investigación. Enseñanza de las Ciencias, 4, 30-35.

GALTON, F.(1889). Natural Inheritance. Londres: MacMillan. https://archive. org/details/naturalinherita01gal tgoog (17 de octubre de 2015)

GIL, D. y MARTÍNEZ-TORREGROSA, J. (1983) A model for problemsolving in accordance with scientific methodology. International Journal of Science Education, 5 (4), 447-455.

HARLEN, W. et al. (2010). Principles and big ideas of science education. Gosport, Hants, UK: Ashford Colour Press Ltd.

MAMLOK-NAAMAN, R. (2011). How can we motivate high school students to study science. Science Education International, 22 (1), 5-17.

MARTÍNEZ AZNAR, T. e IBAÑEZORCAJO, T. (2005). Solving problems in genetics. International Journal of ScienceEducation,27(1),101-121.DOI: 10.1080/09500690410001673801.

MARTÍNEZ-TORREGROSA， J., y VERDÚ, R. (1993). ¿Cómo organizar la enseñanza para un mejor aprendizaje? La estructura de los cursos y los temas en la enseñanza por investigación. Enseñanza de las Ciencias, Número extra, 97-98.

MOMSEN, J. L., LONG, T. M., WYSE, S. A. y EBERT-MAY, D. (2010). Just the facts? Introductory undergraduate 
Biology courses focus on lowlevel cognitive skills. Cell Biology Education-Life Sciences Education, 9, 435-440.

NATIONALCOMMITTEEONSCIENCE EDUCATION STANDARDS AND ASSESSMENT, NRC. (1996). National Science Education Standars. Washington, DC: National Academy. Recuperado de http://www.csun.edu/ science/ref/curriculum/reforms/nses/ nses-complete.pdf [5 de diciembre de 2012].

OSUNA GARCÍA, L., MARTÍNEZ TORREGROSA, J., CARRASCOSA ALÍS, J. y VERDÚ CARBONELL, R. (2007). Planificando la Enseñanza Problematizada: el Ejemplo de la Óptica Geométrica en Educación Secundaria. Enseñanza de las Ciencias, 25 (2), 277-294.

ROCARD, M., CSERMELY, P., JORDE, D., LENZE, D., WALBERG-
HERIKSSON, H. y HEMMO, V. (2007). Science Education Now! A renewed Pedagogy for the Future of Europe. Luxemburgo: Office for Official Publications of the European Communities.

VERDÚ, R. (2004). La estructura problematizada de los temas y cursos de Física y Química como instrumento de mejora de su enseñanza y aprendizaje. (Tesis Doctoral). Departamento de Didáctica de Ciencias Experimentales y Sociales. Universitat de València.

VERHOEFF, R., JAN BOERWINKEL, D. y JAN WAARLO, A. (2009). Genomics in school. EMBO reports, 10 (2), 120-124.

VILLA-GARCÍA, L. Y. y TORRES-ROSERO, M. (2011). Una propuesta para la enseñanza de herencia biológica desde un análisis histórico del concepto. EDUCyT, 4, 116-122. 
Anexo 1

Red de análisis para la valoración de las respuestas al cuestionario ( 0 = no conseguido; 1 = conseguido parcialmente; 2 = conseguido)

Cuestión 1. Acerca de problemas que han podido ser solucionados con conocimientos sobre herencia y que están en el origen del modelo.

(Ver Cuadro 3)

Cuestión 2. Acerca de las teorías de la herencia anteriores o distintas a la de Mendel.

2 El alumno señala teorías sobre la herencia anteriores o distintas (teorías evolucionistas y epigenéticas de la herencia) o la teoría de la herencia de Galton (1889) como mezcla proporcional de caracteres de los progenitores y antepasados.

0 Señala otras teorías biológicas no relacionadas directamente con la herencia de caracteres (teoría de la evolución de Darwin, la de los caracteres adquiridos de Lamarck, fijismo, creacionismo, etc.) o no hace referencia a teoría alguna.

Cuestión 3. Acerca de la utilización con sentido de las hipótesis del modelo de Mendel cuando tratan de explicar a otra persona las ideas importantes sobre la herencia.

2 El alumno expresa los aspectos básicos de la teoría mendeliana (1) que un rasgo físico está controlado por la posesión de al menos dos factores que (2) necesariamente se han de separar y (3) la descendencia sólo recibe uno de cada progenitor; y que tras la fecundación (4) pueden surgir combinaciones de parejas de factores distintas a las paternas, por lo que la expresión final del carácter dependerá de la combinación que se genere y (5) de la relación de dominancia entre ellos.

1 No expresa todos los aspectos anteriores (o lo hacen inadecuadamente), o utiliza directamente un ejemplo concreto con la simbología típica de alelos, sin acompañamiento de explicación abstracta.

0 No se refiere a aspectos básicos concretos de la herencia mendeliana (como cuando expresan sólo que la mitad del ADN proviene del padre y la otra de la madre, que las informaciones genéticas del padre y de la madre "se mezclan") o aluden a términos relacionados (como "genes dominantes y recesivos" o la herencia ligada al sexo) pero sin explicar en ningún caso cómo se transmiten. 
Cuestión 4. Acerca de la planificación de una estrategia para conseguir dos poblaciones con variedades estables de un carácter, a partir de progenitores de genotipo desconocido.

2 El alumno hace referencia a las leyes del modelo para la herencia de un carácter (las indicadas en la cuestión 3, con valoración 2), utilizando la simbología adecuada y explicando con las leyes mendelianas las proporciones que aparecen en la descendencia.

1 O utiliza la simbología mendeliana para un carácter pero sin argumentación alguna, o sólo indica la necesidad de averiguar si son variedades puras y utilizar la autofecundación pero sin desarrollar las leyes mendelianas ni utilizar la simbología característica.

0 O alude a términos y/o metodologías posteriores al modelo, o no utiliza la simbología ni la argumenta adecuadamente, o menciona o identifica la ley de Mendel a la que hace referencia la cuestión pero no la aplica para dar respuesta al problema, o deja la respuesta en blanco.

Cuestión 5. Acerca de la explicación de los resultados de un estudio empírico sobre la herencia de dos caracteres, con desaparición y reaparición de variedades de caracteres en generaciones distintas.

2 El alumno hace referencia a las leyes del modelo para la herencia de dos caracteres (las indicadas en la cuestión 3 puntuación 2 y considerando que (6) la herencia de los factores de un carácter y su expresión no se ven influenciados por la herencia y expresión de otros caracteres), utilizando la simbología adecuada y explicando con las leyes mendelianas las proporciones en la descendencia.

1 Utiliza la simbología mendeliana para dos caracteres pero sin argumentación alguna, o sólo indica la necesidad de aplicar la ley de Mendel relacionada con la herencia de dos caracteres de segregación independiente o la necesidad de parentales dihíbridos, pero sin desarrollar las leyes mendelianas ni utilizar la simbología característica.

0 Alude a términos y/o metodologías posteriores al modelo, o no utiliza la simbología ni la argumenta adecuadamente, o menciona o identifica la ley de Mendel a la que hace referencia la cuestión pero no la aplica para dar respuesta al problema, o deja la respuesta en blanco. 
Cuestión 6. Acerca del conocimiento de las limitaciones del modelo, cuando puede aplicarse (y cuando no) y ver la necesidad de un modelo más amplio.

$2 \mathrm{El}$ alumno hace referencia de forma directa o indirecta al ligamiento de factores o dependencia en la herencia de caracteres, aludiendo a la localización sobre un mismo cromosoma.

1 No hace referencia al ligamento y alude a casos de herencia mendeliana donde las proporciones típicas mendelianas "3:1" y "9:3:3:1" aparecen enmascaradas, como en los casos de codominancia, herencia intermedia, herencia ligada al sexo, genes letales, epistasias u otros casos.

o Piensa que el modelo de herencia mendeliano es universal y se aplica siempre, o indica que no siempre se cumple, sin más, o deja la respuesta en blanco. 
